\title{
A comparison of tactile and blurred visual form perception
}

\author{
PATRICIA APKARIAN-STIELAU \\ Smith-Kettlewell Institute of Visual Sciences, Pacific Medical Center, San Francisco, California 94115
}

and

JACK M. LOOMIS

University of Califormia, Santa Barbara, California $9 \$ 106$

\begin{abstract}
Two experiments were performed to assess the similarity of tactile form perception and visual form perception under conditions of extreme visual blurring. In the first experiment, resolution and relative localization tasks were performed by five subjecis under both tactile and blurred visual presentation. The results obtained here were the same for the two modalities. In the second experiment, all 26 block letters were presented to each of four subjects in two distinct methods of presentation, using both tactile and. blurred visual displays. In one method, the full-field letter was flashed for $1.0 \mathrm{sec}$; in the other, a vertical slit scanned the letter from left to right. For all method comparisons, a strong similarity was found between the patterns of correct responses as a function of letter. In addition, there was a definite similarity between the two modalities in terms of which letters improved in recognizability in the change from the full-field to the slit mode of presentation. However, the overall superiority of the slit method found for tactile recognition was not obtained visually. The two experiments indicate that recognition with blurred vision is similar to recognition using the intact cutaneous sense, although some differences remain.
\end{abstract}

Optical-to-tactile conversion devices such as the Optacon developed by Linvill and Bliss (1966) and the Tactile Vision Substitution System (TVSS) developed by Bach-y-Rita and Collins (Bach-y-Rita, 1972; Collins, 1970) have demonstrated the skin's ability to process pictorial information. These devices provide direct translation of optical images into tactile patterns; this is accomplished by using the video signal from a television camera or a photosensor array to activate a matrix of vibrotactile stimulators. A part from their practical uses, these and similar systems like the Kinotact (Craig, 1974) have proved valuable research tools for investigating tactile pattern perception (Bach-y-Rita, 1972; Craig, 1974; Hill, 1974; Loomis, 1974; Scadden, 1971, 1973; Taenzer, 1972).

In a recent study, Loomis (1974) examined the effects of different modes of stimulus presentation on tactile letter recognition. The 400-point TVSS was

\footnotetext{
The paper is based in part on a thesis submitted by the senior author in partial fulfillment of the requirements for the MA degree at San Francisco State University. The work was supported by Department of Health, Education and Welfare Grant 14-P-55282/9 from the Social and Rehabilitation Services, NIH General Research Support Grant 5S01RR05566, and the Smith-Kettlewell Eye Research Foundation. The authors thank Drs. Paul Bach-y-Rita and Carter C. Collins for the use of the TVSS and Gordon Holmlund and William Hill for their technical assistance. These results were reported at the annual meeting of the Association for Research in Vision and Ophthalmology held at Sarasota, Florida, in May 1975.
}

employed and block capital letters were displayed on the backs of subjects using a total of five different modes of presentation. The recognition results were interpreted in terms of the limited spatial resolution of the cutaneous sense, probably imposed by the neural organization of the lemniscus system with its convergence and divergence of neurons in the ascending pathways and limited cortical representation of most body surfaces, such as the back (Mountcastle, 1961; Werner \& Whitsel, 1973). In particular, the result of slit scanning leading to higher recognition scores than full-field presentation was explicable in terms of limited resolution, for even when the higher spatial frequency content of the stimulus patterns is filtered out in the neural processing, time-varying phase information in the lower spatial frequencies carries the identity of the letter. This result and its interpretation are consistent with the well-known result that tactile localization acuity for a single point, as measured by the error of localization, is considerably finer than the spatial resolution as measured by the two-point limen (Boring, 1930, 1942; Dallenbach, 1932; Jenkins \& Stone, 1941; Weinstein, 1968; Zigler, 1935).

The two present studies were conducted to assess the similarity of the perception of spatial patterns by the intact cutaneous sense and by optically blurred vision. If optically blurred vision were to give detection and recognition results that were identical to those of the cutaneous sense when the stimulus patterns are not degraded, then there would be reason to 
think that the sulact cutaneous sense is functionally equivalent to vision under conditions of extreme blurring.

The first study den,onstrates in a qualitative way that relative localization of a bar stimulus is finer than the resolution of two bar stimuli for both cutaneous perception and blurred vision. The second study compares tactile letter recognition with blurred visual letter recognition under two conditions of stimulus presentation-slit scanning and full-field presentation.

\section{EXPERIMENT I}

\section{Method}

Apparatus. Detailed description of the 400-point TVSS used in 1his experiment can be found elsewhere (Collins, 1970; bach-y-Rita, 1972). Briefly. the system consists of a 20 by 20 matrix of solenoid vibrators mounted on the back of a dental chair, with the spacing between the Teflon-tipped tactors being about $12 \mathrm{~mm}$. The solenoid stimulators, described by Holmlund and Collins (1970), vibrate at $60 \mathrm{~Hz}$ and were set to operate as in the previous study at $10 \mathrm{~dB}$ above threshold $(50 \mathrm{~mW})$. Normally, a television camera is used to drive the tactil. Jisplay; however, in this study. stimuli were generated by means of the toggle switches which permit manual control of each row and column of the display. These switches each have three positions (on, off, and video-controlled), but only the "on" and "otf" positions were used here.

The visual stimuli were produced using the 400-point light display that functions as the visual monitor for the tactile display. A sheet of white Plexiglas was placed in front of the monitor to diffuse the image. The subject then viewed this diffused image monocularly through a pair of strong spherical lenses which served to further blur the image. A detailed description of the apparatus follows.

The visual monitor consists of 400 Sylvania 28ESB tungsten filament bulbs. They are arranged in a square 20 by 20 matrix with a vertical and horizontal spacing (between centers) of $22 \mathrm{~mm}$. A bulb is illuminated when the corresponding tactor is activated by the video signal. When illuminated, each bulb operated at $17 \mathrm{~V}$ and

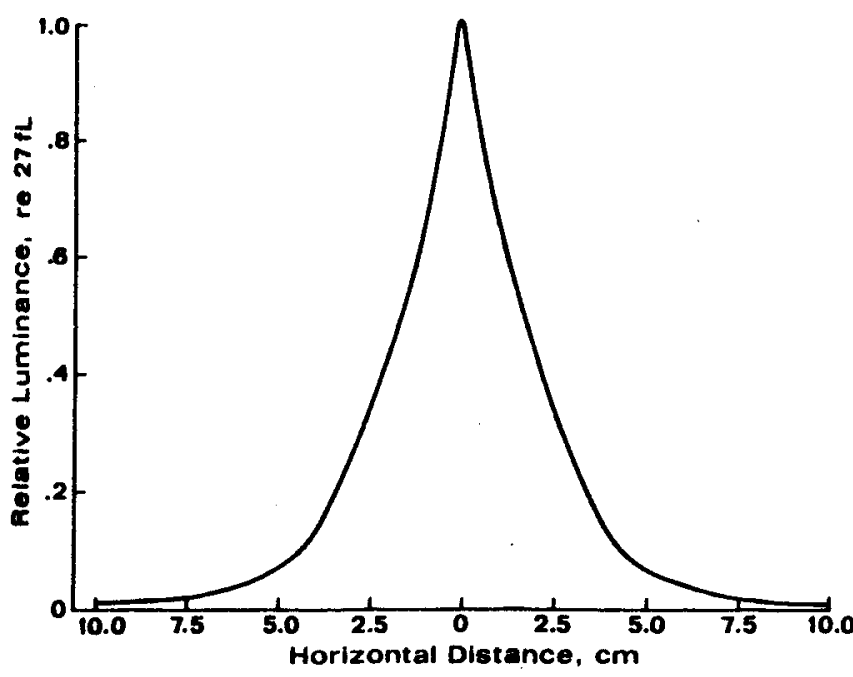

Figure 1. The relative luminance distrbution (In one dimension) along the surface of the diffusing screen when one light point is activated.
$23 \mathrm{~mA}$, which amounts to about $45 \%$ of its rated output. Each bulb is enclosed by a white plastic cover $15 \mathrm{~mm}$ square. The large sheet of white Plexiglas $(6.3 \mathrm{~mm}$ in thickness) was placed in front of the display with a spacing of $6.3 \mathrm{~mm}$ from the surface of the plastic light covers. The relative light output from a single illuminated bulb as measured horizontally across the surface of the Plexiglas, using an SEI photometer, is given in Figure 1 (the peak value corresponds to $27 \mathrm{fL}$ ). With an interbulb spacing of only $22 \mathrm{~mm}$, the diffusing screen alone causes a considerable overlap of the distribution of light from adjacent bulbs.

The observer viewed the light display at a distance of $3.0 \mathrm{~m}$; he wore a pair of ophthalmic trial frames with an occluder in front of the left eye and a pair of lenses plus an aperture in front of the right. The two positive spherical lenses $(12 \mathrm{D}$ and $20 \mathrm{D})$ in effect further defocused the image on the Plexiglas by over $30 \mathrm{D}$. The $3-\mathrm{mm}$ artilicial pupil centered on the tlat surface of one of the lenses served to eliminate changes in the amount of blur with fluctuations in the natural pupil; no changes were observed for the light levels in the display. The room lights were extinguished so that the only effective stimulation came from the light display.

When a single light bulb is illuminated in the visual monitor, the subject sees a large blur circle which is a product of both the diffusing screen and the pair of lenses; it is subjectively uniform in brightness throughout its area, which is bounded by a distinct circumference. We measured the diameter of this subjective blur circle by projecting it onto the field of the left eye when the occluder was removed. Its diameter measured in this way was $6.7^{\circ}$. In comparison with the angular separation of two adjacent points in the light display $\left(25^{\prime}\right)$, the diameter of a subjective blur circle is enormous, the ratio being .063 . Incidentally, the purpose of first blurring the image with a diffusing screen was to eliminate potentially informative striations that were observed in the blur patterns when only the lenses were used.

For complex stimuli, the overall blur pattern on the retina is simply the superposition of the blur patterns of the individual lights in the display that are illuminated. However, because the above description was in terms of the subjective blur circle for a single light, the overall subjective blur pattern can be considered a superposition only if the individual blur circle is truly homogeneous as described and if the visual system is linear. Precise knowledge of the blur pattern is not essential, however, since this paper is concerned with demonstrating only qualitative similarities of blurred vision and cutaneous perception. It is unlikely that one could establish precisely the neural point-spread function (blur distribution for a point stimulus) of the cutaneous sense by matching on the basis of performance to the equivalent optical blur function.

Stimuli. We worked with a basic 4 by 4 submatrix that was positioned midway in height in the full matrix and on the right half, thus falling above the waist on the right half of the subject's back. The corresponding visual stimuli on the monitor were observed by the subject in the manner described above. For the stimuli of the steady condition (the resolution task), two columns or two rows (each four tactors in length) were activated for $1 \mathrm{sec}$ on each trial, with the two alternating columns or rows of the submatrix remaining silent (Figure 2A). For the stimuli of the shift condition (the relative localization task), first one of the four columns was activated for $.5 \mathrm{sec}$ followed immediately by one of the directly adjacent columns to the left or right, also activated for $.5 \mathrm{sec}$ (Figure 2B). There were six of these temporal pairs, one of which was presented on a given trial. The sequences of stimuli in either condition were randomly determined in advance.

Subjects. Five subjects, three female and two male, participated in this experiment as paid volunteers. All have essentially normal vision without correction. Of the tive, two had participated in experiments with the TVSS before.

Procedure. Each subject took part in only one 2-h session, consisting of a total of 96 trials, for each of the following four conditions: steady visual, steady tactile, shift visual, and shift tactile. Conditions were switched in a counterbalanced sequence 


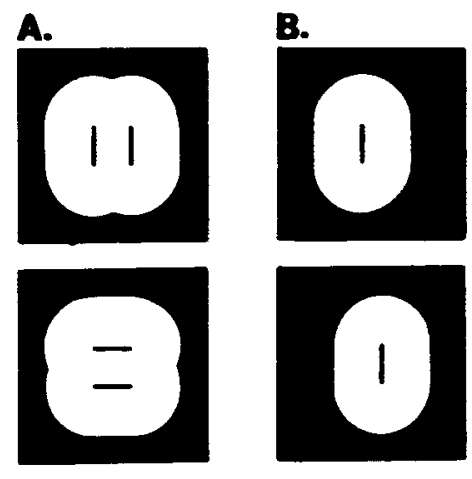

Fure 2. Slanlated bhe patten for the two methed of Brpatment $L$ (A) Twe pals of vertical and bartzontal ban aned $h$ the reodution tack. (B) A typical sequence of stimuli (top to bettom) when the vertical ber is shifted left to right in the relative bealfation unck.

every 24 trials. The subject's task in the steady condition was to say whether vertical or horizontal bars had been presented (a resolution task); the task in the shift condition was to say whether the vertical column had shifted to the left or to the right (a relative localization task). A trial consisted of a warning signal, the stimulus presentation, and the subject's response; no feedback was given until the session was completed.

\section{Results}

The mean values of percent correct responses (averaged over subjects) and their standard errors are shown in Table 1. It is clear that performance in the resolution task (steady) was at chance levels, whereas performance in the relative localization task was nearly perfect. We conclude that subjects were unable to resolve the two spatially separated bars, but could almost always distinguish the direction of a shift of a bar that was moved a distance only half that of the separation used in the resolution task.

\section{Diecusalon}

This short study substantiates with direct evidence what Loomis (1973) had proposed; namely, that a subject can detect a shift of a single target well below the minimal distance corresponding to the spatial resolution. This was expected on the grounds that whereas spatial resolution is limited by the highest spatial frequency transmitted by the sensory system, localization acuity is limited by the sensitivity of the system to phase shifts in the lower spatial frequencies. Although the experiment did not determine explicitly either measure for blurred vision or for the cutaneous sense, it did establish that the relative localization task was performed with near perfect accuracy for shifts only one-half those of the separation used in the resolution task, in which performance was near chance. Some force is given to the visual results in the localization task when one considers that subjects could easily discriminate shifts of the blur circle amounting only to .063 of its diameter $\left(25^{\prime}\right.$ vs. $\left.6.7^{\circ}\right)$.
In general, however, the results are not surprising. It is already well established, both for pressure and for vibrotactile stimuli, that the cutaneous sense is more precise in localizing point targets than in resolving two targets (Boring, 1930. 1942; Dallenbach, 1932; Jenkins \& Stone, 1941; Weinstein, 1968; Zigler, 1935). Likewise, in the case of the visual sense, there is a related ordering of acuity measures; it has long been known that vernier acuity is considerably better than minimum separable acuity (Linksz, 1952; Riggs, 1965; Westheimer, 1972); however, the issue is a bit different in that minimum separable acuity under optimum conditions is limited by the minimum spacing of the foveal cones (Westheimer, 1972) so that localization of a single point presumably would not be much better; vernier acuity is better by virtue of the integrative processes acting on the neural activity produced by the extended lines in the vernier task. The present experiment shows that when the visual acuity is artitically limited by bluring the object, relative localization is much better in analogy to the tactile results.

We suggest that the more precise localization may be a result expected of any system that is characterized by a falloff in sensitivity to the higher spatial frequencies. It may not require the lateral inhibition proposed by von Békésy (1967) to account for his results on tactile localization, particularly those of two points close to one another. This is not to suggest that lateral inhibition is not in fact involved, but merely to suggest that this specific result, the superiority of the localization task, may be accountable for strictly in terms of a high-frequency falloff. Expressed differently, it may not be necessary for the sensory system to "localize" the center of the point spread response (neural "blur circle") by means of funneling; rather, the location of the overall point-spread response may itself be the ultimate meaning of a target's location, thus requiring no funneling process.

The fact that for a system of low spatial resolution a single point can be localized more precisely than two points can be resolved means that patterns which are traced out by a single moving point should be more recognizable than patterns presented all at one time; this assumes, however, no memory limitations nor any prior differential exposure to the two methods of

Table 1

Mean Recognition Accurncies and Standard Erroxs as a Function of Condition

\begin{tabular}{llcc}
\hline & & $\begin{array}{c}\text { Steady } \\
\text { (Percent) }\end{array}$ & $\begin{array}{c}\text { Shift } \\
\text { (Percent) }\end{array}$ \\
\hline \multirow{2}{*}{ Visual } & Mean & 57.8 & 99.4 \\
& SE & 4.6 & .2 \\
Tactile & Mean & 55.6 & 92.8 \\
& SE & 4.0 & 1.1 \\
\hline
\end{tabular}


presentation. The next study, though not using sequential tracing by a point, because we lacked the needed equipment, uses a compromise mode of presentation, scanning with a vertical slit, which is simultaneous in one dimension and sequential in the other. One of the aims of this study was to determine whether, for blurred vision, slit scanning leads to higher recognition accuracy than does full-lield presentation, a finding established for the cutaneous sense by Loonis (1974).

\section{EXPERIMENT II}

\section{Method}

Apparatus. In this study, block capital letters were displayed on the tactile matrix and on the visual monitor, both of which were set to give the same stimulus intensities as in the previous study. A modified television camera was used to drive the displays; normally, the camera is suspended freely on a boom so that the subject can actively scan the environment; however, here the camera was fixed on a tripod, thus removing subject control.

The subject viewed the visual monitor through lenses and diffusing screen, as in Experiment $I$, so that each point on the monitor gave rise to a blur circle of the same angular size and intensity as in that study.

In order to present the letters in both the full-field and slit modes, a simple device was used for displaying the letters to the tripod-mounted camera. It consists of a light and diffuser for back-illumination of the cut-out letters, a slot in front of the diffuser into which a letter card could be inserted, and a movable slot mounted on a motor-driven paper belt into which a vertical slit could be placed for the scanning conditions. The stimuli were 26 cardboard squares, from each of which one of the block capital letters had been cut.

Stimuli. For tactile presentation, the letters were all 18 tactors high, 13 wide, and 2 in stroke width, with the exception of 1 . For visual presentation, each letter was reduced in size to 9 light points high, 7 wide, and 1 in stroke width. The corresponding vertical and horizontal angular dimensions at the eye (seen through lenses and diffuser) are $3.7^{\circ} \times 2.9^{\circ}$ (measuring from the center of the blur circle at one extreme to the center of the blur circle at the other). The overall blur pattern for a $9 \times 7$ letter subtends $9.3^{\circ} \times 8.6^{\circ}$.

Two modes of stimulus presentation were employed for both visual and tactile tasks. In the full-field mode, identical to the
A.
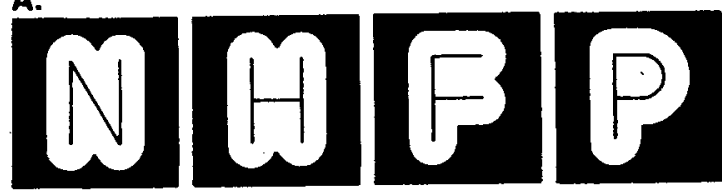

B.
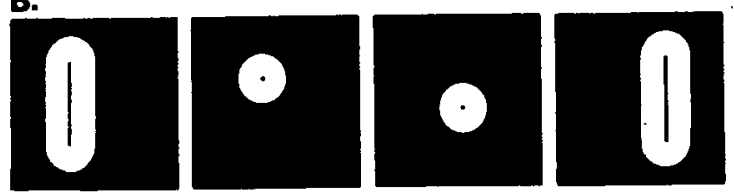

Figure 3. Stmulated blur patterns for selected letton in Experimeat II. These pattern are lea blumed than those actually ween, but they depict only the blur outline without the internal distribution of himinance. (A) The lettew, N, H, F, and $P$ in the visual full-field condition. (B) Four frames from the requential presentation of $\mathbf{N}$ in the visual allt condition.

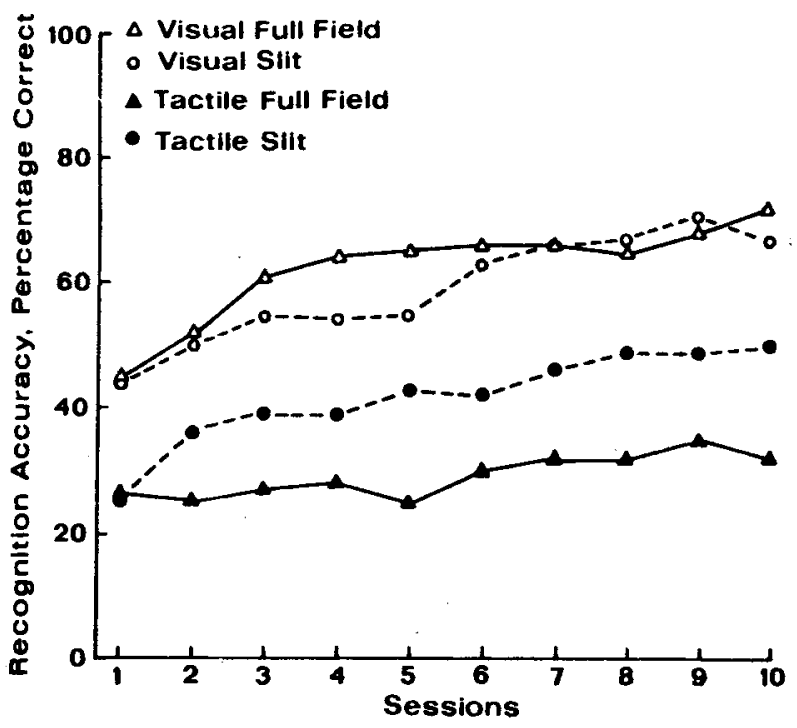

Figure 4. The mean learning curves, averaged over subjects, for each of the four methods of Experiment II.

full-field, stationary-letter mode in the study by Loomis (1974), letters were inserted into the stationary slit and displayed for $1 \mathrm{sec}$. The full-field presentation of the letters, N, H, P, and F as they appear on the diffusing screen is depicted in Figure $3 \mathrm{~A}$. In the other mode, slit presentation, identical to the moving slit, stationary-letter mode of Loomis (1974), a vertical slit was inserted in the movable slot and transported from left to right across the letter such that the entire presentation time (except for 1) was $0.8 \mathrm{sec}$ for both visual and tactile conditions. How the letter $\mathbf{N}$ appears in this mode is depicted in the sequente of four frames in Figure 3B. The full-field mode portrays the letter simultaneously in both the vertical and horizontal directions, whereas the slit mode portrays the information in each vertical section of the letter simultaneously with adjacent sections being portrayed sequentially (and spatially).

Subjects. Two male and two female subjects ranging from 25 to 30 years of age participated in each condition of the experiment as paid volunteers. Three subjects had had no prior experience with the TVSS, while the fourth had participated 2 years previously in the study by Loomis. None of the subjects wore large optical corrections or had any known deficits in visual acuity; in comparison with the blurring of over $30 \mathrm{D}$ in this study, small individual variations are insignificant.

Procedure. All four subjects participated in $102 \cdot \mathrm{h}$ sessions during a period of 10 consecutive weekdays. During each session, the full alphabet was presented twice under each of the four conditions: visual slit, tactile slit. visual full field, and tactile full field. The conditions were alternated on a random schedule every 13 letters, with the sequence of letter presentations also being random. During each trial, the subject was given a warning signal and then the letter presentation. After the subject gave a response, he/she was informed of the correct identity of the letter.

During tactile presentations, subjects were always blindfolded; during visual presentations, subjects wore the trial frames and lenses as described above and fixated the approximate center of the visual display to which they were directed by means of a prior flash of light.

\section{Results}

The recognition accuracies (percent correct responses) averaged over subjects as a function of session are shown in Figure 4. Except for the tactile slit condition, the curves all show clear learning 
Table 2

Mean Recognition Accuracies and Standard Errors as a Function of Condition

\begin{tabular}{llcc}
\hline & & $\begin{array}{c}\text { Full } \\
\text { Field } \\
\text { (Percent) }\end{array}$ & Slit \\
& & (Percent) \\
\hline \multirow{2}{*}{ Visual } & Mean & 67.3 & 67.0 \\
& SE & 3.1 & 3.1 \\
Tactile & Mean & 32.3 & 47.0 \\
& SE & 4.4 & 1.4 \\
\hline
\end{tabular}

trends. The remaining analyses are based on the last five sessions, by which time performance was relatively stable. The mean recognition accuracies for the four conditions averaged over subjects are shown in Table 2 along with the standard errors of the mean. The slit vs. full-field comparisons for both the visual and tactile tasks have each been analyzed by a mixed model analysis of variance (Subjects by Methods) rather than a t test because of large subject variation. For both analyses, subject differences were found to be significant at the .01 level (visual, $F=10.3, \mathrm{df}=$ 3/72; tactile, $F=11.8$, df $=3 / 72$ ).

The primary question being addressed by this experiment is whether the superiority of the slit method found previously in tactile recognition would also be found for blurred vision which was thought to simulate the cutaneous sense. The analysis of variance did not show a significant methods effect for the visual task $(F=.04$, $\mathrm{df}=1 / 3)$, whereas the earlier finding of a difference between methods for the tactile task was replicated $(F=16.8, \mathrm{df}=1 / 3, \mathrm{p}<.05)$. Both analyses revealed significant Subject by Method interactions at the .01 level (visual, $\mathrm{F}=5.5$, $\mathrm{df}=$ $3 / 72$; tactile, $F=5.2, \mathrm{df}=3 / 72$ ).

Because the mean recognition accuracies do not show the full picture, we include the breakdown of recognition accuracy as a function of letter for each of the methods (Figure 5). Several of the analyses to follow are based on these tabulations.

The obvious visual similarity of the patterns of correct responses is corroborated by the positive product-moment correlations between methods. The highest correlation of .81 was obtained between the two slit conditions. The other correlations obtained were: visual full field vs. tactile full field, .65 ; visual full field vs. visual slit, .60; and tactile full field vs. tactile slit, . 75 . All four correlations are significant at the .01 level. These results indicate that letters which are easy (or difficult) to recognize under one condition tend also to be easy (or difficult) to recognize under all conditions.

On the other hand, there are some systematic deviations from this general tendency. Although the average recognition over all letters was the same for both visual modes (slit and full field), some letters were substantially easier under the slit method while others were substantially easier under the full-field mode. The next analysis is an attempt to make some sense of this pattern of difference scores.

In the previous paper, Loomis (1974) divided the alphabet into two groups of letters: Group I (A, H, I, J, L, M, N, T, U, V, W, Y) and Group II (B, C, D, E, $F, G, K, O, P, Q, R, S, X, Z$ ). The Group II letters all have at least two points active within each column of the horizontally scanned presentation, whereas the letters from Group I consist either of solid vertical strokes or a single point within the column. ${ }^{1}$ Group I letters are favored by the slit method because solid strokes or single points are easily registered by the observer, whereas two or three points within a column are not. Slit presentation of Group I letters most closely approximates sequential tracing by a single moving point which, according to Loomis (1974), is limited by localization acuity and not resolution.

We calculated the algebraic difference scores between the corresponding cell values of the slit and full-field conditions for both the visual and tactile tasks. For the visual task, Group I letters showed an average gain of $11.8 \%$, whereas Group II letters showed an average loss of $11.3 \%$ in going from the full-field to slit modes. A t test $(t=2.715, \mathrm{df}=24)$ showed this difference to be significant at the .05 level. Likewise, for the tactile task, Group I letters showed an average gain of $30 \%$ in contrast to the average gain of only $1.6 \%$ for Group II letters. This difference was also significant $(p<.001, t=4.187$, $\mathrm{df}=24)$.

\section{Discussion}

The intent of this study was to learn whether blurred visual recognition would yield results similar to those found with the intact cutaneous sense in the study by Loomis (1974). Loomis had proposed that the tactile performance might be understandable strictly in terms of poor tactile resolution, as if there

\begin{tabular}{|c|c|c|c|c|c|c|c|c|c|c|c|c|c|c|c|c|c|c|c|c|c|c|c|c|c|c|}
\hline \multirow{2}{*}{ ctile Full Field } & & & & & & & & $H$ & & & & & & & & & & & & & & & & & & \\
\hline & 68 & 13 & 33 & 15 & 10 & 45 & 20 & 45 & 98 & 70 & 5 & 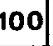 & 3 & 10 & 28 & 60 & 15 & 13 & 10 & 83 & 23 & 20 & 8 & 3 & 25 & 23 \\
\hline & 8 & 15 & 33 & 33 & 20 & 45 & 0 & $5 h$ & & 100 & 3 & 100 & 53 & 33 & 28 & 50 & 8 & 3 & 23 & 88 & 0 & 85 & 65 & 3 & 4 & 4 \\
\hline & 98 & 35 & & & & & 23 & & & 85 & & 100 & 1 & 1 & 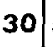 & 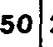 & 20 & 30 & 40 & 100 & 93 & - & 100 & 65 & $83 / 9$ & . \\
\hline & & 13 & 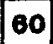 & & & & 45 & lon & & & 55 & 98 & 23 & & 10 & 15 & 28 & 53 & & & 30 & 83 & 55 & $88 \mid$ & 689 & 95 \\
\hline
\end{tabular}

Figure 5. The percentage of correct repponese, averaged over subjecto, as a function of letter and method of presentation. 
were a low-pass spatial filter between the stimulus and the recognition process. The blurring operations of this experiment in effect introduced a low-pass filter between the letters and the visual system. Using blurred vision as a simulation of the intact cutaneous sense seemed promising, since a given person's decision processes during recognition and response selection were potentially the same for both tasks; had we attempted a simulation using a formal model of recognition and low-pass filtering, we would have needed to specify explicitly these recognition and response processes. The results show that two of the comparisons between the two modalities support the limited-resolution hypothesis for tactile form perception and the third does not, although at possible reason for this failure will be given in what follows.

The strongest evidence for a similarity between blurred vision and tactile perception derives from the modestly high correlations between the patterns of correct responses for the four conditions. The correlation between visual slit and tactile slit is an impressively high .81 , with the other correlations equal to at least .60 . That the correlation between the two full-field modes is only .65 may either be a consequence of our failure to match the two conditions more closely in terms of "equivalent blur" or may indicate that some other factor, such as the enormous prior visual experience with spatial displays, must be taken into account. These significant positive correlations indicate that the blurring operation does simulate an aspect of tactile form perception, for without blurring, all letters would be recognized visually all or most of the time, leading to near-zero correlations between the two modalities.

The other result which supports the limitedresolution hypothesis is the analysis of the letter-specitic difference scores. Each difference score was obtained by subtracting the recognition accuracy for a given letter in the full-field mode (averaged over subjects) from the corresponding value for the slit mode. In the tactile task, most of these difference scores are positive, but some are substantially larger than others. In the visual task, the gains balance the losses to give an overall equal performance in the two conditions. What is interesting is that these patterns of difference scores match to some extent the dichotony of the alphabet suggested by Loomis (1974), with the Group I letters being those which are most nearly traced out by the vertical scanning slit. For both the tactile and visual comparisons, Group I letters have significantly higher gain scores than Group 11 letters. Inasmuch as some letters are already highly recognizable in the full-field conditions, a closer examination of the difference scores shows the strength of this trend. In the tactile task, when the letters I, $L$, and $T$ are exluded because of their already high recognition accuracies $(98,100,83)$, Group I letters show a mean increase of $39.2 \%$; this contrasts with an average increase of $1.6 \%$ for Group II letters.
When we examine the same letters in the visual task. many of the Group I letters are already so high in recognizability in the full-field mode that increases are impossible, or nearly so. Of the remaining letters that could show much of an increase $(H, M, N, W$, $Y$ ), all except $N$ show a sizeable gain, in contrast to the average decrease of $11.3 \%$ for the letters of Group II.

The one result which failed to come out as predicted was the finding that the visual slit mode led to essentially the same overall recognition accuracy as did the visual full-field mode. This differs from the tactile result obtained clearly both in the present study and in the previous study by Loomis (1974), that slit scanning leads to higher tactile recognition accuracy than does full-field presentation. The visual result appears to present a paradox, for slit scanning under conditions of blurring does convey more information than full-field presentation, since the effects of blurring are then limited only to the vertical dimension (within the two columns of stimulation). Rather than taking this result as disconfirming the limited-resolution hypothesis for cutaneous perception. we suggest that some other factor favoring the visual full-field mode must have offset the expected theoretical advantage of the slit mode.

The most likely candidate for this factor is the differential amount of exposure subjects have had to the two modes of presentation; considering that subjects have had a lifetime of experience in interpreting spatial displays but virtually none in interpreting scanned displays, it is not surprising that at least in the tirst half of training, the full-field mode had a substantial advantage. Perhaps we would have observed an eventual superiority of the slit mode with considerably more practice than the subjects received here.

More specifically, the way in which familiarity with full-field presentation would be advantageous here is probably in the subject's optimal utilization of the lower spatial frequency content of the letters. Sighted people clearly have learned to recognize visual patterns when only the lowest (relative) spatial frequency content is available, as, for example, when objects or persons are at a great distance. That people do this has been demonstrated by Harmon (1973, p. 78) in a study of visual recognition of human faces under conditions of extreme blurring. This is not to say that the lower spatial frequencies are not being used by the cutaneous sense, for clearly, by our hypothesis, these are the only frequencies not being liltered out in the neural processing. Rather, it is that the visual system makes more optimal use of the same information by virtue of prior experience. To this extent, the limited-resolution hypothesis may be insufficient to explain the difference between visual and tactile form perception.

In retrospect, we could have insured that slit scanning would have led to superior recognition pertormance over full-lield presentation had we used 
forms that were all equated in terms of their lower spatial frequency content (of the same overall shape) but were different in terms of their higher frequencies. If these equated lower frequencies were those just transmitted by the optical filter (the blurring operation) and the equivalent neural filter of the cutaneous sense, then the forms in the full-field mode would all be indistinguishable, leading to chance performance for both modalities. However, since the slit scanning mode is not resolution-limited in the horizontal dimension, as demonstrated by the results of Experiment I, some information would be transmitted by this mode of presentation, allowing some improvement in performance for both modalities.

Although it may seem, from what we have said, that sequential presentation, either scanning with a linear slit or tracing with a point, should allow one to bypass in large measure the effects of a low-pass spatial filter, there remains the very real limitation of memory. Whether the subject simply registers and holds in memory all of the absolute positions stimulated by the scanning point during the presentation or whether he/she registers and stores the relative shifts of the point, the subject could be expected to identify a pattern, which in the full-field mode is only a large blur, if his/her memory capacity is sufficient. For relatively simple patterns, like letters, memory capacity would not be exceeded, so that the superiority of the sequential mode would be expected; however, for complex patterns, the greater amount of information available in the sequential mode would be balanced or outweighed by the losses of information from memory, resulting in performance equal or inferior to that under full-field presentation.

\section{REFERENCES}

BACh-y-Rrta, P. Brain mechanisms in sensony substitution. New York: Academic Press, 1972.

Békésy, G. von. Sensory inhibition. Princeton, N.J: Princeton University Press, 1967.

Boring, E. G. The two-point limen and the error of localization. American Journal of Psychology. 1930, 42, 446-449.

Boning, E. G. Sensation and perception in the history of experimental psychology. New York: Appleton-Century-Crofts, 1942.

Colsuns, C. C. Tactile television-mechanical and electrical image projection. Institute of Electrical and Electronics Engineers Transactions on Man-Machine Systems, 1970, MMS-11, 65-71. Craic, J. C. Pictorial and abstract cutaneous displays. In F. A. Geldard (Ed.). Cutaneous communication systems and devices. Austin, Tex: Psychonomic Society, 1974.
Dallenbach, K. M. A comparative study of the error of localization on the fingertips. American Joumal of Psychology, 1932. 44. 327-331.

Harmon. L. D. The recognition of faces. Scientific American, 1973. 229. 70-82.

HrLL, J. W. Limited field of view in reading lettershapes with the fingers. In F. A. Geldard (Ed.), Cutaneous communicution systems and devices. Austin, Tex: Psychonomic Society. 1974.

Holmlund, G. W.. \& Collins. C. C. An electromagnetic tactile stimulator. Joumal of Biomedical Systems, 1970. 1. 25-40.

Jenkins, W. L., \& Stone, L. J. Recent research in cutaneous sensitivity. II. Touch and the neural basis of the skin senses. Psychological Bulletin. 1941, 38, 69-91.

Linksz. A. Physiology of the eye. (Vol. 2) Vision. New York: Grune \& Stratton. 1952.

Linvili. J. G., \& Bliss, J. C. A direct translation reading aid for the blind. Proceedings of the Institute of Electrical and Elictronics Engineers. 1966, 54, 40-51.

Loomis. J. M. Tactile letter recognition under different modes of stimulus presentation. Perception \& Psychophysics, 1974, 16. 401-408.

Mountcastle. V. B. Some functional properties of the somatic afterent system. In W. A. Rosenblith (Ed.), Sensory communication. Cambridge, Mass: M.I.T. Press, 1961.

RigGs, L. A. Visual acuity. In C. H. Graham (Ed.), Vision and visual perception. New York: Wiley, 1965.

Scadde i, L. A. Perception of patterned vibratory stimulation: An evaluation of the Tactile Vision Substitution System. Doctoral thesis, University of the Pacific, 1971.

SCAdDEN, L. A. Tactile pattern recognition and body loci. Perception, 1973, 2, 333-336.

TAENZER. J. C. An information processing model for visual and tactual reading. Perception, 1972, 1, 147-160.

Weinstein. S. Intensive and extensive aspects of tactile sensitivity as a function of body part, sex and laterality. In D. R. Kenshalo (Ed.), The skin senses. Springfield. IIl: Thomas, 1968.

WERner, G., \& Whitsel. B. L. Functional organization of the somatosensory cortex. In A. Iggo (Ed.), Handbook of sensory physiology (Vol. II), Somatosensory system. New York: Springer-Veriag, 1973.

WESTHEIMER, G. Visual acuity and spatial modulation thresholds. In D. Jameson \& L. M. Hurvich (Eds.), Handbook of sensory physiology (Vol. 7/4), Visual psychophysics. Berlin: Springer-Verlag. 1972.

ZigleR, M. J. The experimental relation of the two-point limen to the error of localization. Jourmal of General Psychology, 1935, 13, 316-331.

\section{NOTE}

1. The only exception to this rule is the letter A, which through half of its presentation is represented by only a single point in each column and through the other half, by two points. The letter $J$ falis into Group 1, for it did not have a horizontal stroke on top.

(Received for publication May 19, 1975; revision received August 11, 1975.) 